\title{
Make the right measurement - discovery of an allosteric inhibition site for p300-HAT
}

Anna S. Gardberg, Jonathan Wilson, Annissa J. Huhn, Archana Reddy, Florence Poy, Jeremy Setser, Valerie Vivat, and Francois Brucelle

Constellation Pharmaceuticals, Cambridge, MA

Histone acetyltransferases (HATs) and histone deacetylases (HDACs) catalyze the dynamic and reversible acetylation of proteins, an epigenetic regulatory mechanism associated with multiple cancers. Indeed, HDAC inhibitors are already approved in the clinic. The HAT paralogs p300 and CREB-binding protein (CBP) have been implicated in human pathological conditions including several hematological malignancies and androgen receptor-positive prostate cancer. Others have reported CoA-competitive inhibitors of p300 and CBP with cell-based activity. Here we describe 2 compounds, CPI-0489076 and CPI-0671090, discovered through p300-HAT HTS screening, which inhibit p300-HAT via binding at an allosteric site. We present the high resolution (1.72 and $2.32 \AA$ ) co-crystal structures of these molecules bound to a previously undescribed allosteric site of p300-HAT. 